\title{
Research on Functional Design of Power Information and Communication Scheduling Intensive Support System
}

\author{
Fangfang Dang ${ }^{1, a}$, ,Huiping Meng ${ }^{1, b}$,Shiwen Wang ${ }^{1, c}$, You Sun ${ }^{1, d}$ \\ ${ }^{1}$ Information \& Telecommunication Co. of State Grid Henan Electric Power Company, \\ Zhengzhou 450052 China

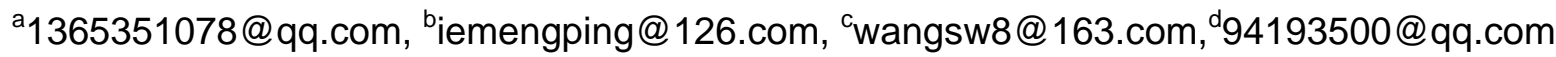

Keywords: Information and communication; Intensive scheduling; Support system;Centralized network management

\begin{abstract}
In the process of comprehensive promoting the electric power enterprise information and communication provincial, city and county "integration, specialization, lean" construction, the scheduling "integrated" support system can't fully meet the needs of intensive development of information and communication scheduling. In order to improve the functions of provincial information and communication scheduling system, adjust and optimize the scheduling support system architecture, this paper presents a information and communication intensive scheduling support system which is based on"data acquisition layer, data management layer, business application layer" and many elaborate methods to realize intensive scheduling from aspects of the scheduling management, operation and maintenance management, visualization, centralized scheduling platform, centralized network management and support system. Finally, the paper conducts some functional optimization design about the support system to improve the efficiency and security of the information and communication network steadily.
\end{abstract}

\section{Introduction}

The information and communication network of power, which is an important part of strong and smart grid, is an important foundation of the protection of production,operation,management and supply of services. With the development of information and communication network, the supportive role in safeguarding of information and communication scheduling is becoming more and more prominent. It is urgent to build the provincial information and communication intensive scheduling mode which is based on "one scheduling, two maintenance, integrated operation and maintenance" ${ }^{\text {[1] }}$. Unified and efficient information and communication system can effectively solve the problems about the structural lack of operation and maintenance staff, the large number of equipment maintenance and the differences of business processes between regions. While the construction of the information and communication intensive scheduling support system, which as an ancillary system, not only gradually improves the level of information and communication professional intensive, flat, professional management, but also enhances the ability to support the power production safety and business management efficiency.

\section{Status and Problems}

\section{Status of information and communication scheduling support system}

Information and communication scheduling support system is an accurate and efficient tool which is able to meet the ordinary management needs of information and communication scheduling and deal with emergency events occurred during the operation in real time.Through the operation on the support system in various functional modules, dispatchers can realize the whole process control about such as command, maintenance, operation mode etc. They can also rationally and scientifically allocate the information and communication resources and initially realize the information and communication professional "unified command and control, unified device 
monitoring, unified defect treatment, unified maintenance management, unified resource way management". Currently, the enterprise has built IMS, ISS, TMS, IDS, IRS and other support systems, while in accordance with the ideas of "centralized platform, business integration, decisionmaking intelligent, advanced and practical", these systems have been integrated into the "integrated information and communication scheduling operation support platform"(referred I6000), all of these have realized the automated monitoring, process management, interactive display and intelligent decision on the information and communication scheduling.

\section{Problems of information and communication scheduling support system}

As the scale of information and communication networks growing rapidly, the role of information and communication scheduling support system has become increasingly prominent. However, there are still some weaknesses, mainly in the following aspects.(1) Accuracy: there are many data problems such as data source dispersion, difficult to access heterogeneous databases and the complexity of large data interface, which may bring statistical data inconsistencies, data dependency disorder, non-standard data formats and incomplete data. These issues will lead to the presence of dirty data and affect the accuracy of the information system.(2)Efficiency: some business designs of the existing information and communication scheduling support system are unreasonable. For instance, the vertical and horizontal integration about the method tickets and maintenance tickets is not perfect and affect the efficiency of the scheduling work.(3) Real-time: the existing data acquisition interface does not fully meet the demand of real-time data acquisition of new equipment and impact real-time data of the support system.(4)Visibility: in the existing information and communication system , there are still some deficiencies to improve in support of the information and communication networks, equipment of engine room, operation of business systems and real-time display of indicators.(5) Security: due to the expansion of the scope of supervision, dispatchers need to quickly sort and filter the current alarms and supervise the latest dynamic of alarm handling form centralized pages. While the existing filtering function of alarm handling mechanism is weak and it has brought some potential problems on the security of information and communication networks ${ }^{[2][3]}$.

\section{Model Design}

\section{Overall architecture design}

In order to meet the expansion of information and communication network and coverage of business,the increase of content management and the improving of requirements, it is urgent to optimize the functionality of existing systems which emphasis on real-time monitoring, resource methods, command and disposal, statistical analysis, horizontal interconnect and so on. Information and communication scheduling intensive support system adopts the hierarchical design which is based on "high cohesion, low coupling". The low layer is composed of some classes and related components and provides services to the top layer through a unified and transparent interface. The top layer applies for the services of lower layer through the interface and does't need to consider the details of underlying implementation.This mechanism can realize the separation between layers and reduce the coupling procedure[4]. The system consists of business application layer, data management layer, data acquisition layer, horizontal interface and vertical interface. The overall system architecture as is shown in figure 1: 


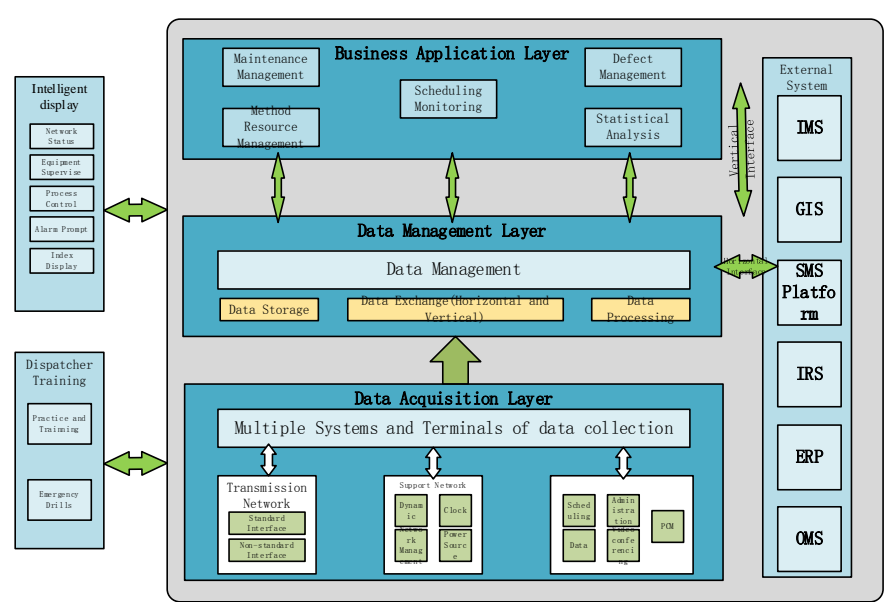

Fig 1: Scheduling intensive support system model design

(1) business application layer

In accordance with the target of information and communication scheduling intensive, the support system should complete the closed-loop process form citizen companies to provincial companies on scheduling monitoring, defect management, maintenance management, resource methods and statistical analysis and so on. All of these will realize the provincial concentration about operation and maintenance management, defect management, resource allocation and statistical analysis.

(2) data management layer

This layer mainly include some functional modules such as data modeling, security management, system management, graphics engine, process engine, service bus, report form management, data storage and data interconnection .It aims to complete all kinds of data storage and exchange and provide reliable data support for business applications.

(3) data acquisition layer

This layer is composed of data acquisition and intelligent control system. It can complete the data acquisition of transmission network(standard, non-standard), supporting network (power environment,clock synchronized network,etc.) and business network(dispatching exchange, integrated data network, administrative exchange, video conference, PCM, etc.) through the related interfaces.

(4) horizontal interface

This part can realize the basic data sharing, process interoperability and interactive applications with external systems (IMS, GIS, ERP, etc.) through the standard data interface. It also can unify the data source of information and communication, eliminate the data barriers between systems and strengthen the integration of function and business of information and communication.

(5) vertical interface

The support system collects the data of transmission network, supporting network and business network through data acquisition interface which is located in the bottom of the system. While the vertical interface can conduct the data cleaning, storage, exchange and processing in the data management layer and finally to provide a reliable data source for maintenance, resource management and scheduling of the business application layer. It can also realize data sharing,process interoperability and interactive applications to improve the accuracy and availability of the system.

\section{Function realization of scheduling intensive support system}

The functional design mainly involves scheduling management, support system operations management, intelligent display, the dispatcher training, centralized transmission network management, support network and business network system[5], as is shown in figure 2. 


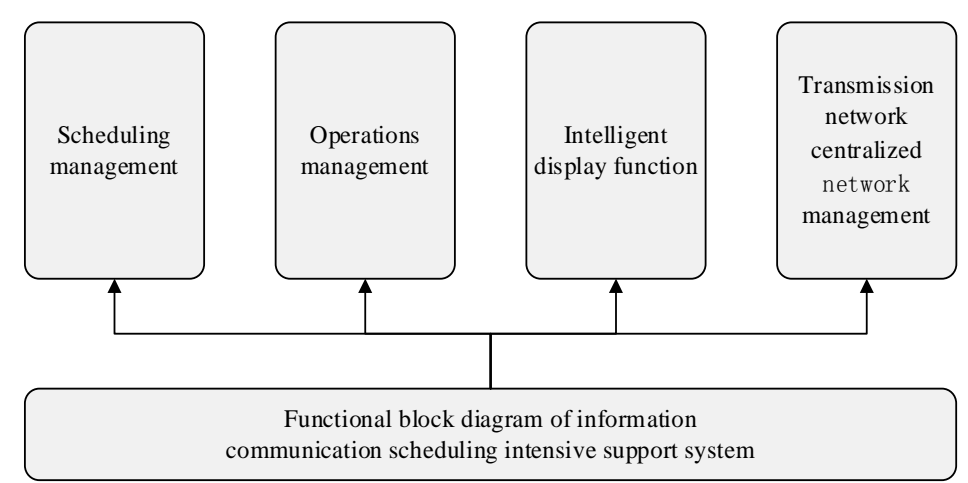

Fig 2: Functional block diagram of information communication scheduling intensive support system

\section{Scheduling management}

Scheduling management implements the work ticket management, duty management, alarm management, defect management, maintenance management, method manage and other functions. This module will complete the closed-loop process form citizen companies to provincial companies on scheduling business realize the provincial concentration about operation and maintenance management, defect management, resource allocation and statistical analysis.

(1) The work order management

After the intention of provincial information and communication scheduling, the quantity of work orders will increase greatly. In order to convenient the dispatchers to pay close attention to the work orders during the duty, the support system should provide customized query about the related orders on duty.

(2)Duty management

With the increase of the number of centralized scheduling attendants, support systems should provide flexible scheduling management functions to strengthen the management of information and communication scheduling attendants. On the basis of the existing management of scheduling, this module can provide duty-switch function which achieves the interconnection with the existing SMS platform to make sure that every attendant can get the SMS alert before going to work.

(3) Alarm management

Due to the extension of regulatory scope, scheduling attendant need to sort and filter the current alarm fast and supervise the current situation of alarms from the centralized monitoring page. Support system has the function of the centralized display for multi-level communication network alarms. It also can improve the mechanism of the alarm and start defect repair order after identified the defective equipment.

(4) Defect management

Defect repair order must be linked with equipments and the number of defects should be analyzed according to the time of occurrence, subordinate units, the type of equipment, defect reasons and so on.

(5) Maintenance management

This module provide the function of adjusting the process of repair order that the equipment maintenance process of provincial level and above maintains the status quo, the equipment maintenance process of prefecture-level was checked in regional and signed after being submitted to the provincial scheduling organization. Its open and completed application is the same with level 3 maintenance process of communications equipment .

(6) Methods manage

This module provide the function of adjusting the process of methods and business application. The communication methods at provincial level and above maintains the status quo, while the communication methods at prefecture-level was written and checked in regional and carried out after being submitted to the provincial information and telecommunation company. 
Operations management

Operations management should satisfy the requirement of the scheduling after scheduling intensive and the provincial centralized of operations management of systems. In the process of operations, it is crucial to meet two aspects of timeliness and standardization of data. Operations management functional requirements as is shown below.

(1) Acquisition and monitoring

The purpose of this design is to meet the existing data acquisition interface of transmission network, support network and so on. It will make a specification of interface to support existing the acquisition of new equipment.For the data which can not be collected automatically, the method of manual control or bulk import may provide supplements.

(2) Alarm disposal

This module implements the monitor of automatic alarm about transmission network, business network and support network. It will give a root alarm notification after a variety of handling from collecting the alarm to alarm threshold, alarm compression, correlation analysis and so on and can query other alarm information through the correlation of root alarm. At least, the ways of alarm should include dynamic graphics, text tips, voice prompt, reserved SMS and E-mail reminders.

(3) Query and statistics

This module has the function of classified query, in the same way, the results should have figure, table display effect according to their characteristics and can be exported to standard format (Excel spreadsheet). All kinds of queries support fuzzy query and exact query. For the business data, it is necessary to have a variety of query statistic entrances which is classified by system, station,business and time section.

Intelligent display function

Intelligent display function mainly completes the centralized display of the information and communication network, equipment, business system operation status and real-time index of information and communication company of provinces, cities and company. It includes real-time index display, network operation status display, alarm display, process display, etc.

(1) the network operation status display

Collect the real-time data of provincial intensive dispatching system and display the results hierarchically in figure and table.

(2) Alarm display

Allow users to choose the display range flexibly such as selection area,type of equipment, network system, alarm category and so on after obtaining the permission.

(3) Process display

Display the processes of method ticket, repairment ticket and defect ticket, urge and identify them according to the set time.

Transmission network centralized network management

According to the scale of area network, management system and the difference of technical ability, the centralized mode of network management system can be divided into the following three kinds.

(1) "1 + N" mode

"1 + N" mode is consisted of a provincial network management center and $\mathrm{N}$ prefecture network management center. It supports the weak centralized network management model through the "terminal" counter loop and has the capacity of centralized network monitoring at the provincial level.

(2) " $1+0 "$ mode

" $1+0$ " mode sets up the unified network management center in the provincial company. It is capable of supporting the mode of strong centralized network management and monitoring the centralized network at the provincial level. At last, it can meet requirements of disaster recovery. 
(3) "1 + X" mode

"1 + X" mode can be named as "partial centralized". According to different dimension of concentration, $X$ refers to the number of prefecture-level companies that don' $t$ need to be centralized form the point of administration. While from the perspective of network manufacturers, $\mathrm{X}$ can refers to the number of equipments and network management systems that don' $t$ need to be centralized.

\section{Conclusions}

The construction of information and communication scheduling intensive system at the provincial level is an effective measure to enhance the level of professional management of information and communication. The design of power information and communication scheduling intensive support system is based on multi-level architecture and realize the data sharing of information and communication. This design transfers the system operation mode from dispersed,local and single way to the integrated, intensive operation way. Under the new situation and organization, it will improve the efficiency of information and communication dispatching work and provide a strong support for the work to develop .

\section{References}

[1] Jinpei Lu. Intensive distribution network scheduling and technical support system[J].Intelligent Rural Network. 2012,06:38-40 .

[2]Hongbo Lv.Research on power integrated management mode regulation[J].China Southern Power Grid Technology[D].North China Electric Power University,2011.

[3] Xiquan Li. Hunan Power Scheduling PDCA management mode[D]. Hunan University,2011.

[4] Linsheng Xu.Design and Implementation Based on Data Mining Power Dispatching Management System [D].Electronic Science and Technology University of ZheJiang, 2011.

[5] Junfeng Yao. Data storage strategy under intensive modes of production scheduling energy metering platform[J].Power and Energy.2013,123-124. 